\title{
Quantum Return Probability for Substitution Potentials
}

\author{
César R. de Oliveira*and Giancarlo Q. Pellegrino ${ }^{\dagger}$ \\ Departamento de Matemática - UFSCar, São Carlos, SP, 13560-970 Brazil
}

September 3, 2018

\begin{abstract}
We propose an effective exponent ruling the algebraic decay of the average quantum return probability for discrete Schrödinger operators. We compute it for some non-periodic substitution potentials with different degrees of randomness, and do not find a complete qualitative agreement with the spectral type of the substitution sequences themselves, i.e., more random the sequence smaller such exponent.
\end{abstract}

PACS numbers: 05.45.Pq, 02.30.-f, 71.30.+h, 71.55.Jv

*e-mail: oliveira@dm.ufscar.br

†e-mail: gian@dm.ufscar.br 
Anomalous transport in non-periodic structures is due to intricate quantum interferences which may also lead to localization of wave functions. Another possibility is ballistic motion, mainly related to periodic structures. Here we consider transport properties in nearest-neighbours tight-binding models in $\mathbb{Z}$ whose general Hamiltonian $H$ is given by

$$
(H \psi)_{n}=\psi_{n+1}+\psi_{n-1}+\lambda V_{n} \psi_{n},
$$

with $\lambda>0$ and potentials $V=\left(V_{n}\right)_{n \in \mathbb{Z}}$ generated by some non-periodic substitution sequences.

Among the characterizations of (de)localization and transport we single out the (average) moments of the "position" operator

$$
m_{\alpha}(T)=\frac{1}{T} \int_{0}^{T} d t \sum_{n=-\infty}^{\infty}\left|n-n_{0}\right|^{\alpha}\left|\psi_{n}(t)\right|^{2}, \quad \alpha \neq 0,
$$

and the (average) return probability

$$
C(T)=\frac{1}{T} \int_{0}^{T} d t\left|\psi_{n_{0}}(t)\right|^{2} .
$$

In relations (2) and (5) it is implicitly assumed that the initial condition is $\psi_{n}=\delta_{n, n_{0}}$. Both quantities $m_{\alpha}(T)$ and $C(T)$ have strong physical appeal and in some cases are attainable to theoretical and numerical investigations. We notice that the return probability was one of the first quantities considered in the seminal paper by Anderson on localization in disordered structures [1].

It has been found that for large $T$ [2, 3, 田, 5, 6, , 7]

$$
m_{\alpha}(T) \sim T^{\alpha \beta(\alpha)} \text { and } C(T) \sim T^{-\Delta} .
$$

Localization should be characterized by vanishing exponents $\beta$ and $\Delta$, ballistic motion by $\beta(\alpha)=\Delta=1$, while anomalous transport by $0<\beta, \Delta<1$. Notice that $\beta(2)$ is related to the direct conductivity via the anomalous Drude formula [8, 9].

In this work we consider potentials $V$ in (I) generated by some substitution sequences and compute the exponent for the decay of the return probability as a function of the degree of randomness of those sequences. As it will 
be seen later, in most cases $\Delta$ can not be obtained directly from numerical integration of the time-dependent Schrödinger equation; namely, the standard fitting procedure given by equation (4) works only for the well-investigated case of Fibonacci potentials (to be defined below). We then propose an alternative approach, based on the energy spectral decomposition of the initial quantum state, which not only is able to retrieve the known Fibonacci results but permits us to exhibit an effective exponent also for other non-periodic substitution potentials such as Thue-Morse, Rudin-Shapiro, paperfolding and period doubling (see below for their precise descriptions). The main conclusion will be that there is no perfect correspondence of the exponent ruling the algebraic decay of the return probability and the degree of randomness of the own substitution sequences, as one expects based on general considerations (i.e., more random the sequence smaller such exponent). Now we proceed to the details of the points just outlined, including a justification for the choice of $\Delta$ as our exponent of interest.

The RAGE theorem and Wiener lemma give direct physical meaning to the standard types of spectra, i.e., point and continuous (absolutely and singular) in the sense that one of their corollaries is that for continuous spectra $\beta(\alpha)>0$ and $\Delta>0$, while for point spectra $\Delta=0$; it is left room for $\beta>0$ even for point spectra due to the tail of eigenfunctions and domain intricateness [10, 11]. The determination of such exponents is a quantitative step from RAGE and Wiener lemma which, by its turn, is still related to deep spectral quantities, i.e., generalized dimensions of the (positive) spectral measures associated to the initial state $\psi$. It has been rigorously proven that $\beta(\alpha)$ is bounded from below by the information dimension $D_{1}$ [2], 3] (all dimensions are related to the corresponding spectral measure) and also conjectured that $\beta(2) \approx D_{0}$ ( $D_{0}$ denotes the fractal dimension of the spectrum). In an interesting paper Guarneri and Mantica [12] have presented examples of homogeneous fractal spectral measures, i.e., with generalized dimensions $D_{q}=D_{0}$ for any $q$, for which $\beta(\alpha)$ is not constant and no simple exact relation seems to hold between the thermodynamics of the spectrum and the 
exponent $\beta$, so that "multiscaling does not require multifractality" [12]; this was called quantum intermitency in [12, 13]. See [14] for some recent results on a particular class of systems and other references.

On the other hand, it was rigorously proven [5, 6] that the exponent $\Delta$ ruling the algebraic decay of the return probability equals the correlation dimension $D_{2}$. We note that such relation supposes the limit defining the dimension $D_{2}$ does exist. See also [4] where this relation was first proposed in the context of anomalous diffusion. Therefore we have selected the return probability, its corresponding exponent $\Delta$ and correlation dimension $D_{2}$, as the main tools for analyzing our systems.

Relevant examples of anomalous diffusion are generated by almost-periodic potentials $V$; an important class of such potentials is given by $V$ induced by non-periodic (primitive) substitution sequences [15, 16]. These sequences form a convenient laboratory for the study of anomalous transport since in all rigorously analyzed cases they generate singular continuous spectra for the tight-binding model (1), although the own spectral types of substitution sequences are not equal; for example, Fibonacci (FCC), paperfolding (PF) and period doubling (PD) substitution sequences have point autocorrelation measures, Thue-Morse (TM) has singular continuous autocorrelation measure, and the autocorrelation measure of Rudin-Shapiro (RS) substitution sequence is absolutely continuous. Although all these sequences are almost periodic, their spectral properties characterize them qualitatively from "ordered to random" since periodic and quasiperiodic sequences have pure point autocorrelation measures (as FCC, PF and PD do), whereas independent random sequences have absolutely continuous autocorrelation measures (as $\mathrm{RS}$ does). The TM sequence lies in an intermediate place. It is also worth noting that all these sequences give rise to strictly ergodic dynamical systems with zero topological and generalized entropy [15, 17].

Due to different degrees of randomness of the substitution sequences, it was expected differences in the spectral properties of the corresponding tightbinding Hamiltonians (1), but as already commented above all rigorously 
studied cases have presented singular continuous spectrum [18, 19, 20, 21, 22. 23] (the RS case is an important open problem [19, 22, 24]; the PF case is also open). Our main goal in this Letter is to investigate whether the spectral character of the sequence generating the potential is responsible for different physics through details of the return probability behaviour. To this end we consider potentials $V$ in (1) generated by the five non-periodic sequences FCC, PF, PD, TM and RS. $\Delta$ can be computed either from numerical integration of the time-dependent Schrödinger equation, and then fitting a straight line on $\log C(t) \times \log t$, or directly computing $D_{2}$ from its definition (which also involves a straight line fitting - see below). However, as already anticipated, we have faced problems in linear fittings in both procedures (except for the well-investigated case of FCC potentials) and we propose a pragmatic approach to get such exponents which is able to recover the FCC known results.

Now we present the rules describing the sequences we use to generate $V$. FCC, PD and TM sequences are constructed with an alphabet of two letters $\{a, b\}$ through the substitutions

$$
\begin{gathered}
a \rightarrow a b, b \rightarrow a(\mathrm{Fcc}), \quad a \rightarrow a b, b \rightarrow b a \quad(\mathrm{TM}), \\
a \rightarrow a b, b \rightarrow a a(\mathrm{PD}) .
\end{gathered}
$$

Beginning with $a$ and applying successively the substitution rules, non-periodic sequences are obtained; e.g., the Thue-Morse sequence is given by

\section{abbabaabbaababba...}

The RS and PF sequences can be obtained with an alphabet of four letters $\left\{a^{\prime}, b^{\prime}, c^{\prime}, d^{\prime}\right\}$, the substitutions

$$
\begin{aligned}
& a^{\prime} \rightarrow a^{\prime} b^{\prime}, \quad b^{\prime} \rightarrow a^{\prime} c^{\prime}, \quad c^{\prime} \rightarrow d^{\prime} b^{\prime}, \quad d^{\prime} \rightarrow d^{\prime} c^{\prime} \quad(\mathrm{RS}), \\
& a^{\prime} \rightarrow a^{\prime} b^{\prime}, \quad b^{\prime} \rightarrow c^{\prime} b^{\prime}, \quad c^{\prime} \rightarrow a^{\prime} d^{\prime}, \quad d^{\prime} \rightarrow c^{\prime} d^{\prime} \quad(\mathrm{PF}),
\end{aligned}
$$


and then the identifications $a^{\prime}, b^{\prime} \rightarrow a$ and $c^{\prime}, d^{\prime} \rightarrow b$ in both cases; the first elements of the RS sequence are

\section{aaabaabaaaabbb...}

We then use these substitution sequences to define our potentials $V$; we take $V_{n}=0$ if the $n$-th letter of the sequence is $a$ and $V_{n}=1$ in case it is $b$. There are standard ways to extend the potential for negative values of $n$ [20, 22], but we avoid such issue by taking a finite sample of $N$ sites, with $n \geq 0$, and using the initial wavefunction $\psi_{n}=\delta_{N / 2, n_{0}}$. In this way we construct the almost-periodic substitution potentials $\lambda V$ and investigate $\Delta$ as function of the degree of randomness of the potential and its intensity $\lambda$.

It is known that FCC, PD and TM generate potentials whose spectra of (1) are singular continuous for all $\lambda \neq 0$. The case of RS has been numerically investigated in [25, 26] indicating point spectrum for $\lambda>2$ and mixed spectrum, i.e., point and singular continuous, for $0<\lambda \leq 2$ (notice we use a scale for the potential values which is different from [25]). For Hamiltonian (11) with PF potential it is only known that its spectrum has no absolutely continuous component, since it is primitive [22]; from a rigorous point of view the lack/presence of eigenvalues in this case is also an open question.

The case of FCC Hamiltonian has also been considered in [4] and a good agreement between the value of $\Delta$ from numerical integration of Schrödinger equation and $D_{2}$ was found. Let's recall the definition of $D_{2}$ associated to a spectral measure $\mu$ and how it is usually estimated [4, 6]. For $\varepsilon>0$ let $B_{\varepsilon}(x)$ denote the open ball of centre $x$ and radius $\varepsilon / 2$ and set

$$
\gamma(\varepsilon)=\int \mu\left(B_{\varepsilon}(x)\right) d \mu(x)
$$

the correlation dimension of $\mu$ is given by the limit

$$
D_{2}=\lim _{\varepsilon \rightarrow 0} \frac{\log \gamma(\varepsilon)}{\log \varepsilon} .
$$


If this limit does not exist one defines $D_{2}^{+}$and $D_{2}^{-}$via limsup and liminf, respectively. The latter remark is important here since we have found numerical indications that the limit in the definition (5) does not exist for general substitution potentials. In numerical practice we have a finite basis approximation for (1) whose spectrum is composed of eigenvalues $\chi_{k}$; then we divide the energy range into boxes $B_{j}$ of length $\varepsilon$, approximate

$$
\gamma(\varepsilon) \approx \gamma^{*}(\varepsilon)=\sum_{j}\left(\sum_{\chi_{k} \in B_{j}}\left|a_{k}\right|^{2}\right)^{2}
$$

and get $D_{2}$ from the linear fitting of $\log \gamma^{*}(\varepsilon) \times \log \varepsilon . a_{k}$ is the projection of the initial wavefunction $\psi$ on the eigenvector with eigenvalue $\chi_{k}$. We have used these procedures to recover $D_{2}$ and $\Delta$ found in [4] for the FCC case as illustrated in figure 1. However such techniques do not work for substitution potentials distinct from FCC (as far as we have checked), since no clear region with linear behavior is found in the plots $\log \gamma^{*}(\varepsilon) \times \log \varepsilon$ and $\log C(t) \times \log t$, as exemplified in figure 2 for the PD potential with $\lambda=1$.6. We suspect this behaviour is an indication that the limits defining the scale exponents $D_{2}$ and $\Delta$ are not well defined in such situations; then we propose a pragmatic approach to extract effective exponents $D_{2}$ by selecting a particular value $\varepsilon^{*}$ of $\varepsilon$. Before presenting our approach we stress we have also tried to get well-defined exponents by site averaging on samples beginning at locations $0,1 \times 10^{4}, 2 \times 10^{4}, \cdots, 5 \times 10^{4}$, but quite similar behaviours were found.

We begin our argument with the remark that if $\varepsilon$ is smaller than the least eigenvalue spacing (we just ignore the possibility of degenerate eigenvalues in this argument) then

$$
\gamma^{*}(\varepsilon)=\sum_{k}\left|a_{k}\right|^{4}
$$

which resembles the so-called inverse participation ratio (which does not depend on $\varepsilon$ ); this also gives a physical interpretation for $D_{2}$. As a naive first guess for an effective exponent one could try to use $\sum_{k}\left|a_{k}\right|^{4}$ instead of $\gamma^{*}(\varepsilon)$, but the exact value of $\varepsilon$ to be used in an approximation to (5) is not clear at all. The theoretical determination of $D_{2}$ involves the limit $\varepsilon \rightarrow 0$; 
finite basis approximations preclude this limit and also too small values of $\varepsilon$ are meaningless, despite the inverse participation ratio interpretation. For sufficient small values of $\varepsilon$ we have

$$
\frac{\gamma^{*}(\varepsilon)}{\sum_{k}\left|a_{k}\right|^{4}} \approx 1 ;
$$

we suggest to pick $\varepsilon^{*}$ as the smallest value of $\varepsilon$ such that the l.h.s. of (7) considerably deviates from 1 , so still keeping track of $\gamma^{*}(\varepsilon)$ and also the inverse participation ratio interpretation in operation. Then we estimate the effective $D_{2}$ as $D_{2}^{*}$ given by

$$
D_{2}^{*}=\frac{\log \gamma^{*}\left(\varepsilon^{*}\right)}{\log \varepsilon^{*}} .
$$

Let's be more precise on how we have picked up $\varepsilon^{*}$ in practice. By using double precision (16 digits) in our code, we adopted that after diagonalization we can numerically resolve the spectral quantities with 8 digits, i.e., half of the number of digits of the code precision, so that $\varepsilon^{*}$ is given by the smallest value of $\varepsilon$ such that $\gamma^{*}(\varepsilon)-\sum_{k}\left|a_{k}\right|^{4} \geq 10^{-8}$ or, equivalently, the smallest $\varepsilon$ such that

$$
\left|\frac{\gamma^{*}(\varepsilon)}{\sum_{k}\left|a_{k}\right|^{4}}-1\right| \geq \frac{10^{-8}}{\sum_{k}\left|a_{k}\right|^{4}} .
$$

We remark that in most cases $\varepsilon^{*}$ can also be obtained directly from visual inspection, as in figure $2 \mathrm{~b}$, and the precise value $10^{-8}$ is not so relevant since in general $\gamma^{*}(\varepsilon)$ has a pronounced jump at $\varepsilon=\varepsilon^{*}$.

We have tested our approach in the FCC case and have got very good agreement with the computed values of $D_{2}$ from our linear fittings and the values reported in 团. In figure $2 \mathrm{~b}$ we show a typical curve used to estimate $\varepsilon^{*}$, and in figure 3 we compare the values of the exponents $\Delta$ and $D_{2}$ as calculated in figure 1 and also the matching values of $D_{2}^{*}$ from equation (8) for the FCC case. From now on we use this procedure to estimate the exponents $D_{2}^{*}$ for the other substitution Hamiltonians (1) considered here.

Its now time to discuss our numerical results and details of their implementations. The return probability $C(T)$ was calculated by direct diagonalization of the Hamiltonian and eigenfunction expansion of the initial state; 
we used bases of size $N \approx 1 \times 10^{3}$ and checked some results with bases of size $N \approx 2 \times 10^{3}$. The initial condition was always concentrated on the centre of the basis $n_{0}$ and we have followed its time evolution until time $T_{f}$ for which the modulus of the amplitude at one of the border sites reaches $1 \times 10^{-6}$. For the calculation of $D_{2}$ we have considered subdivisions of the spectrum in subintervals of size $\varepsilon$ ranging from the least eigenvalues spacing (we disregarded multiple eigenvalues) up to $10^{-2}$. We could seldom conceive a linear behaviour in such $\log -\log$ plots in both $D_{2}$ and $\Delta$ cases in order to extract faithful exponents, so that we were left with the task of finding $\varepsilon^{*}$ and computing only $D_{2}^{*}$.

In figure 4 we present a summary of our main numerical results, i.e., the values of $D_{2}^{*}$ for some substitution potentials as function of the potential intensity $\lambda$. Since we are not aware of any complete rigorous spectral classification for Hamiltonian (1) in the cases of RS and PF sequences, we have also used $T_{f}$ as indication of any possible (de)localization transition; this is the reason for the restriction of the RS case to $\lambda \leq 1.7$; for all substitution sequences we have found $T_{f} \leq 10^{3}$ for $\lambda \leq 2$, but for RS $T_{f}$ jumps from $T_{f} \approx 10^{3}$ for $\lambda=1.7$ to $T_{f} \approx 10^{5}$ for $\lambda=1.8$, which characterizes absence (at least numerically) of extended states. If non-localized states are present their "amounts" suffer a drastic reduction at $\lambda \approx 1.8$ so that we have not detected them. Recall that in [25, 26] it is argued that all states of RS Hamiltonian should be localized for $\lambda>2.0$.

Since no such sharp transition in $T_{f}$ was found for the PF Hamiltonian, its values of $D_{2}^{*}$ are close to the corresponding values for $\mathrm{PD}$, and both substitution sequences have point autocorrelation measures, we conjecture

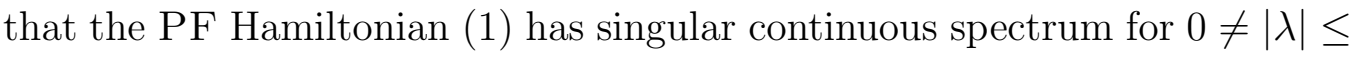
2 (maybe also for any $\lambda \neq 0$ ) as PD Hamiltonian does [18].

Besides the above conjecture we see from figure 4 that for all sequences the exponent $D_{2}^{*}$ decreases as $\lambda$ increases (as physically expected). Since different exponents were found despite the proven singular continuous spectra of FCC, TM and PD Hamiltonians, we see that $D_{2}^{*}$ is able to discern these 
operators. The values of $D_{2}^{*}$ for the RS are very close to the TM case, but in principle one would not expect this since the autocorrelation measure of the RS sequence is Lebesgue measure (the same for random sequences). Notice that the spectral classification of the underlying sequence generating the potential does not reflect exactly in $D_{2}^{*}$, since these values for PF and PD are below the corresponding ones for TM and RS, the latter sequences being considered "more random" than the former ones. Notice, however, that for the most popular sequences FCC, TM and RS we have found agreements with the classification through the sequence spectral type, i.e., this order implying decreasing values of $D_{2}^{*}$.

The higher values of the exponents for RS compared to PD indicates that the presence of extended states mixed with numerically found localized states [25, 26] does not necessarily imply lower exponents $D_{2}^{*}$.

Summing up, we have found that for substitution potentials in general the dynamical exponent $\Delta$ and the correlation dimension $D_{2}$ are difficult to be obtained from direct linear fittings (at least with the basis sizes we used; we suspect this is a consequence of the quantum intermitency and multiscaling in time of the dynamics [12, 13]) and we have proposed $D_{2}^{*}$ as an effective exponent, which has recovered $D_{2}$ in the cases it can be directly obtained. We then computed $D_{2}^{*}$ for some substitution potentials and have not found a complete qualitative agreement with the spectral type of the substitution sequences themselves, i.e., more random the sequence smaller $D_{2}$. Only for $\mathrm{RS}$ we have got indications of a spectral transition from extended (critical) to localized states, although its values of $D_{2}^{*}$ are higher than those for PD and PF cases.

\section{Acknowledgments}

CRO was partially supported by CNPq (Brazil); discussions with U. Grimm at the Max Planck Institute for the Physics of Complex Systems (Dresden, Germany) are acknowledged. GQP thanks the support by FAPESP (Brazil). 


\section{References}

[1] Anderson P W 1958 Phys. Rev. 1091492

[2] Guarneri I 1993 Europhys. Lett. 21729

[3] Guarneri I and Mantica G 1994 Ann. Inst. H. Poincaré A61 369

[4] Ketzmerick R, Petschel G and Geisel T 1992 Phys. Rev. Lett. 69695

[5] Holschneider M 1994 Commun. Math. Phys. 160457

[6] Barbaroux J-M, Combes J-M and Montcho R 1997 J. Math. Anal. Appl. 213698

[7] Last Y 1996 J. Funct. Anal. 142402

[8] Schulz-Baldes H and Bellissard J 1998 Rev. Math. Phys. 101

[9] Schulz-Baldes H and Bellissard J 1998 J. Stat. Phys. 91991

[10] del Rio R, Jitomirskaya S, Last Y and Simon B 1995 Phys. Rev. Lett. 75117

[11] del Rio R, Jitomirskaya S, Last Y and Simon B 1996 J. Anal. Math. 69 153

[12] Guarneri I and Mantica G 1994 Phys. Rev. Lett. 733379

[13] Mantica G 1997 Physica D 109113

[14] Barbaroux J-M and Schultz-Baldes H 1999 Anomalous quantum transport in presence of self-similar spectra (Technishe Univ. Berlin, preprint)

[15] Queffélec M 1987 Substitution Dynamical Systems-Spectral Analysis, Lecture Notes in Mathematics Vol. 1294 (Berlin: Springer)

[16] Axel F and Gratias D (eds.) 1995 Beyond Quasicrystals (Berlin: Les Editions de Physique and Springer Verlag) 
[17] Berthé V 1994 J. Phys. A: Math. Gen. 277993

[18] Bellissard J, Bovier A and Ghez J-M 1991 Commun. Math. Phys. 135 379

[19] Bovier A and Ghez J-M 1993 Commun. Math. Phys. 15845

[20] Bovier A and Ghez J-M 1993 Commun. Math. Phys. 166431

[21] Sütő A 1989 J. Stat. Phys. 56 525

[22] Hof A, Knill O and Simon B 1995 Commun. Math. Phys. 174149

[23] Hörnquist M and Magnus J 1995 J Phys A: Math. Gen. 28479

[24] Allouche J-P 1997 J. Math. Phys. 381843

[25] Dulea M, Johansson M and Riklund R 1992 Phys. Rev. B 45105

[26] Dulea M, Johansson M and Riklund R 1993 Phys. Rev. B 478547 


\section{Figure Captions}

\section{Figure 1}

a) Log-log (base 10) of the return probability (dashed line) $C(t)$ for the FCC potential with $\lambda=1.0$ versus time. The slope of the straight line fitting (full line) corresponding to $\Delta$ is indicated.

(b) Log-log (base 10) of $\gamma^{*}$ (dashed line) for the FCC potential with $\lambda=1.0$ versus $\varepsilon$. The slope of the straight line fitting (full line) corresponding to $D_{2}$ is indicated.

\section{Figure 2}

Same as in figure 1 but for the PD potential with $\lambda=1.6$. The arrow in $\mathrm{b}$ ) indicates $\varepsilon^{*}$; the first point at left in b) corresponds to the least eigenvalue spacing, for which $\gamma *=\sum_{k}\left|a_{k}\right|^{4}$. No linear fitting is shown.

\section{Figure 3}

Scaling exponents $D_{2}, D_{2}^{*}$ and $\Delta$ for various potential intensities $\lambda$ of the FCC potential.

\section{Figure 4}

Effective scaling exponents $D_{2}^{*}$ as function of $\lambda$ for some substitution potentials. 


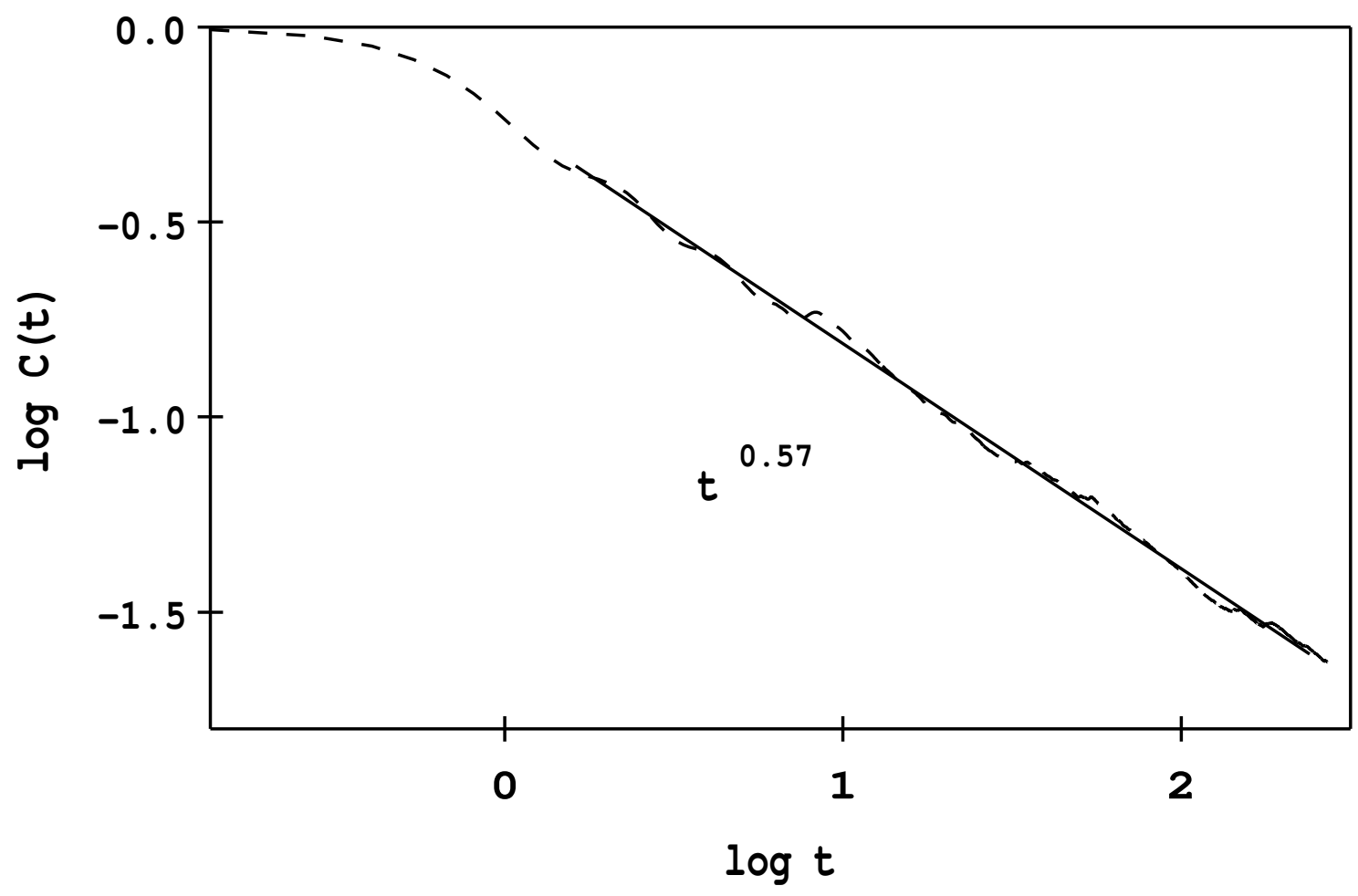

Figure 1a 


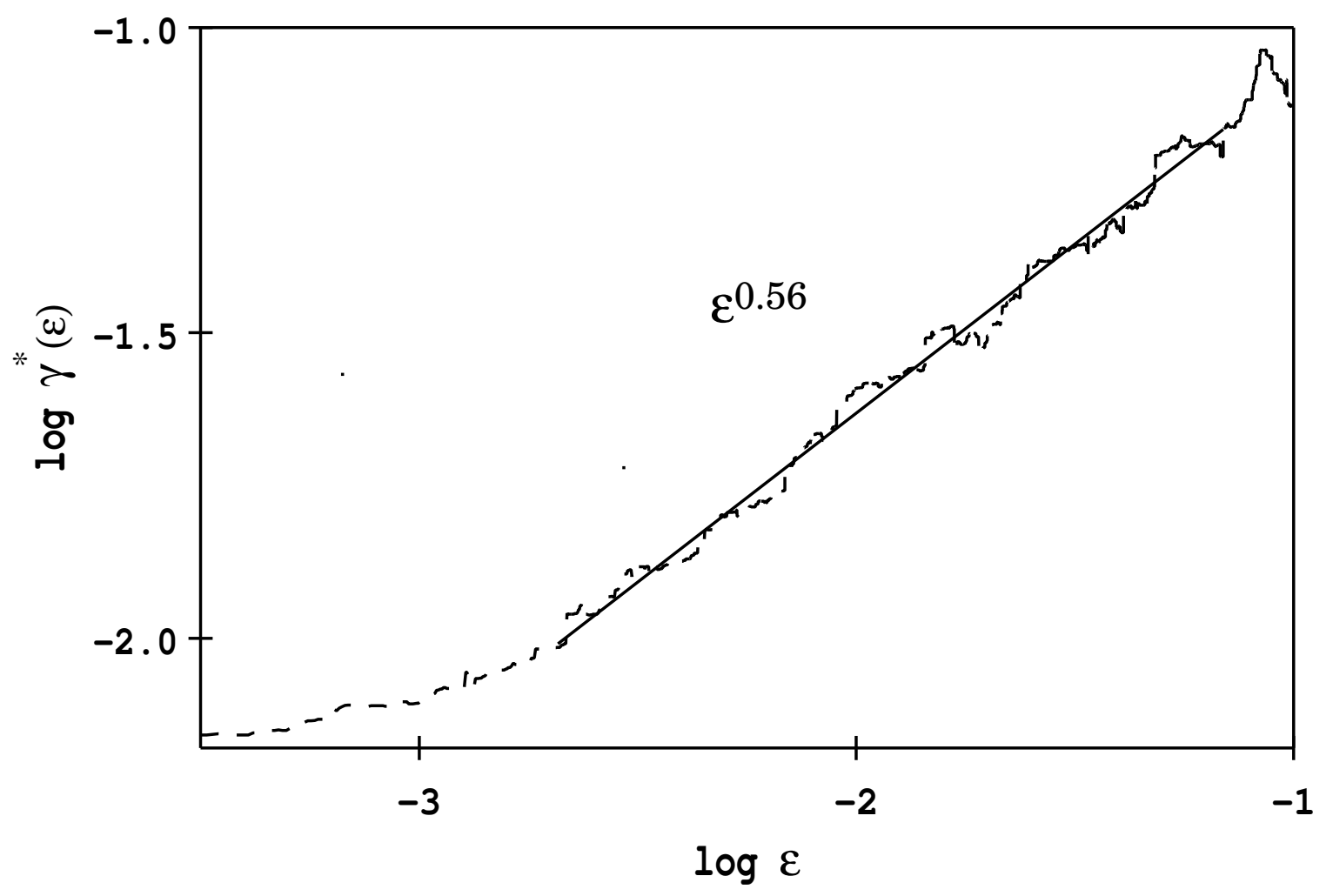

Figure 1b 


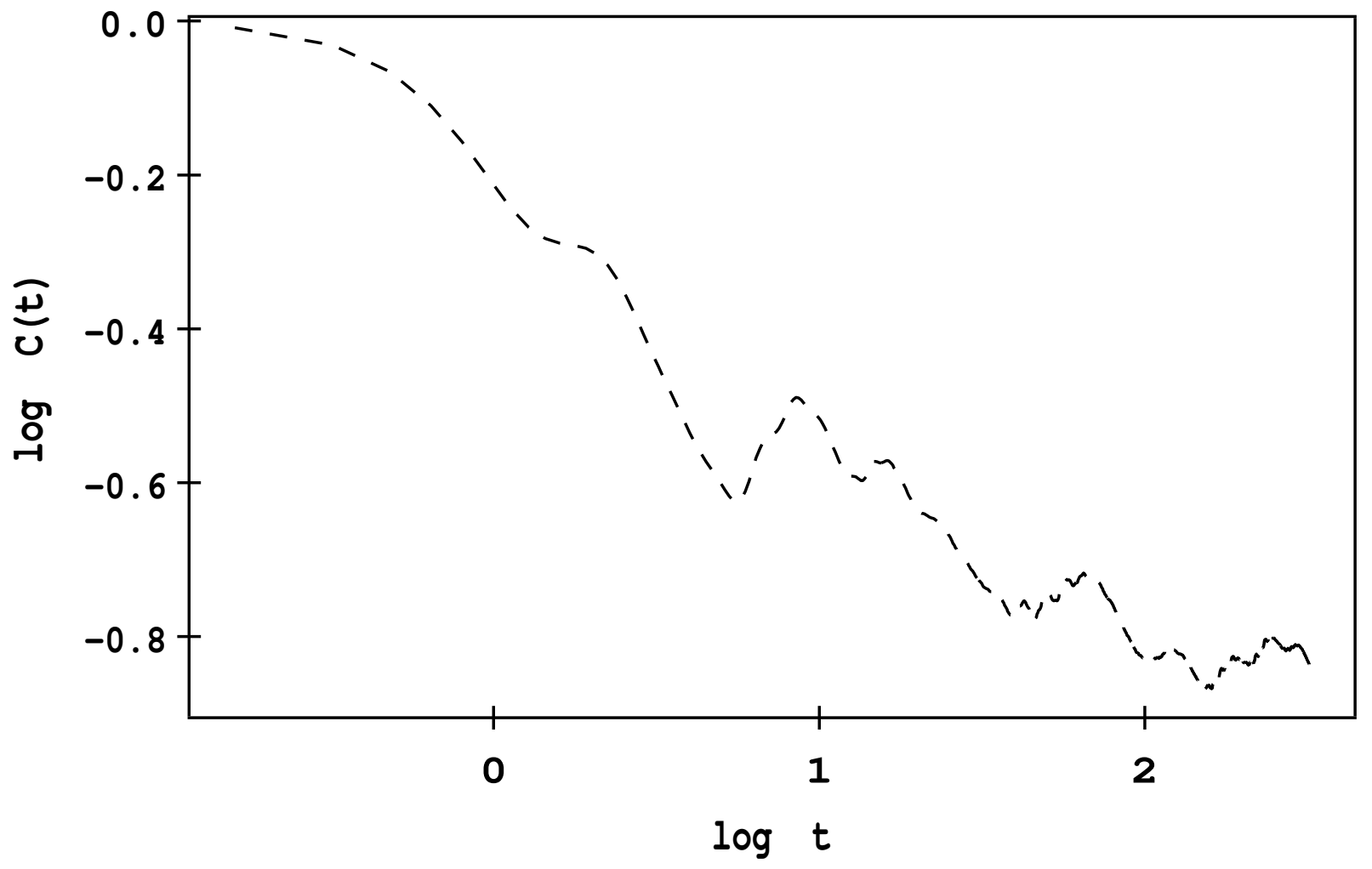

Figure $2 a$ 


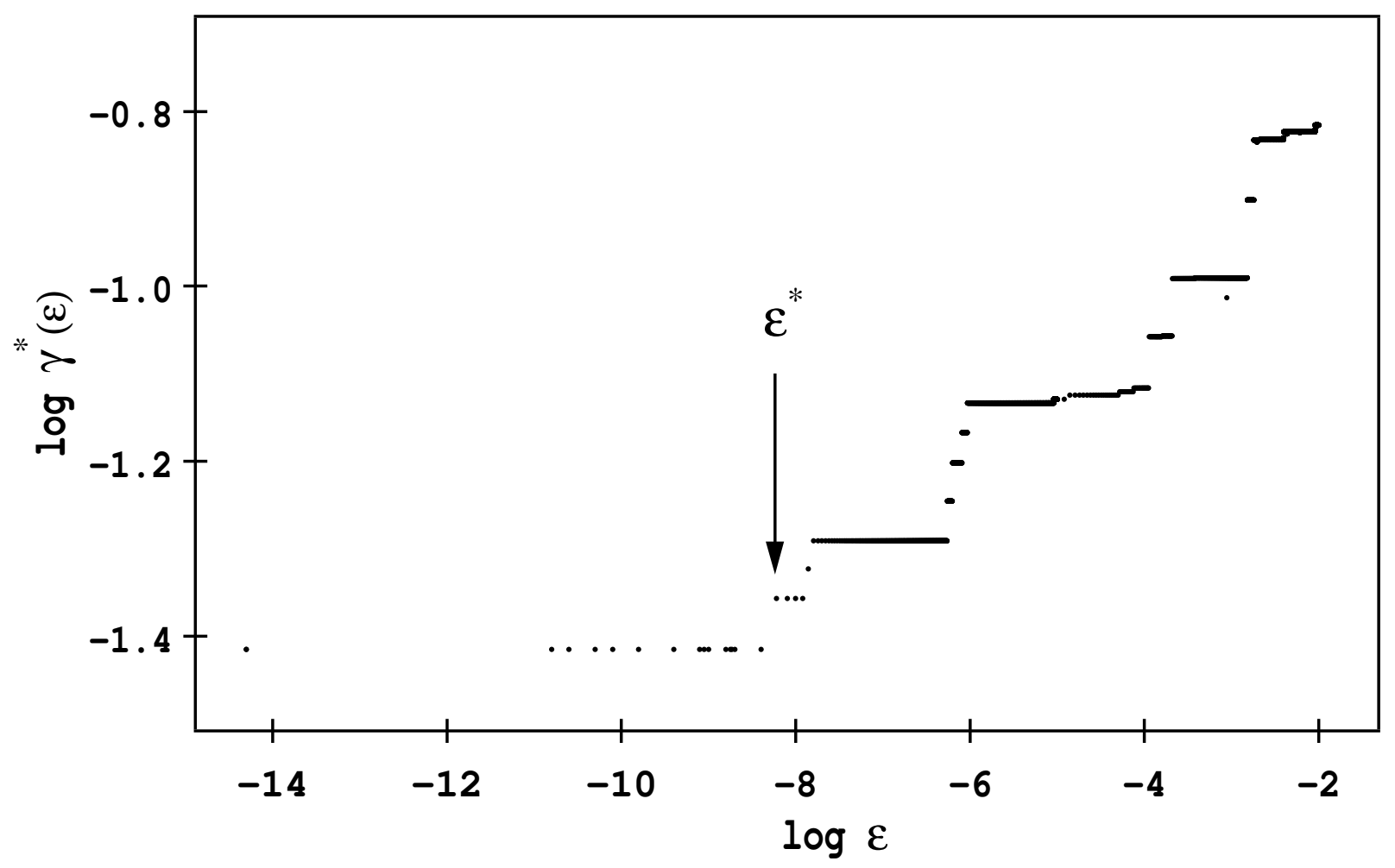

Figure $2 b$ 


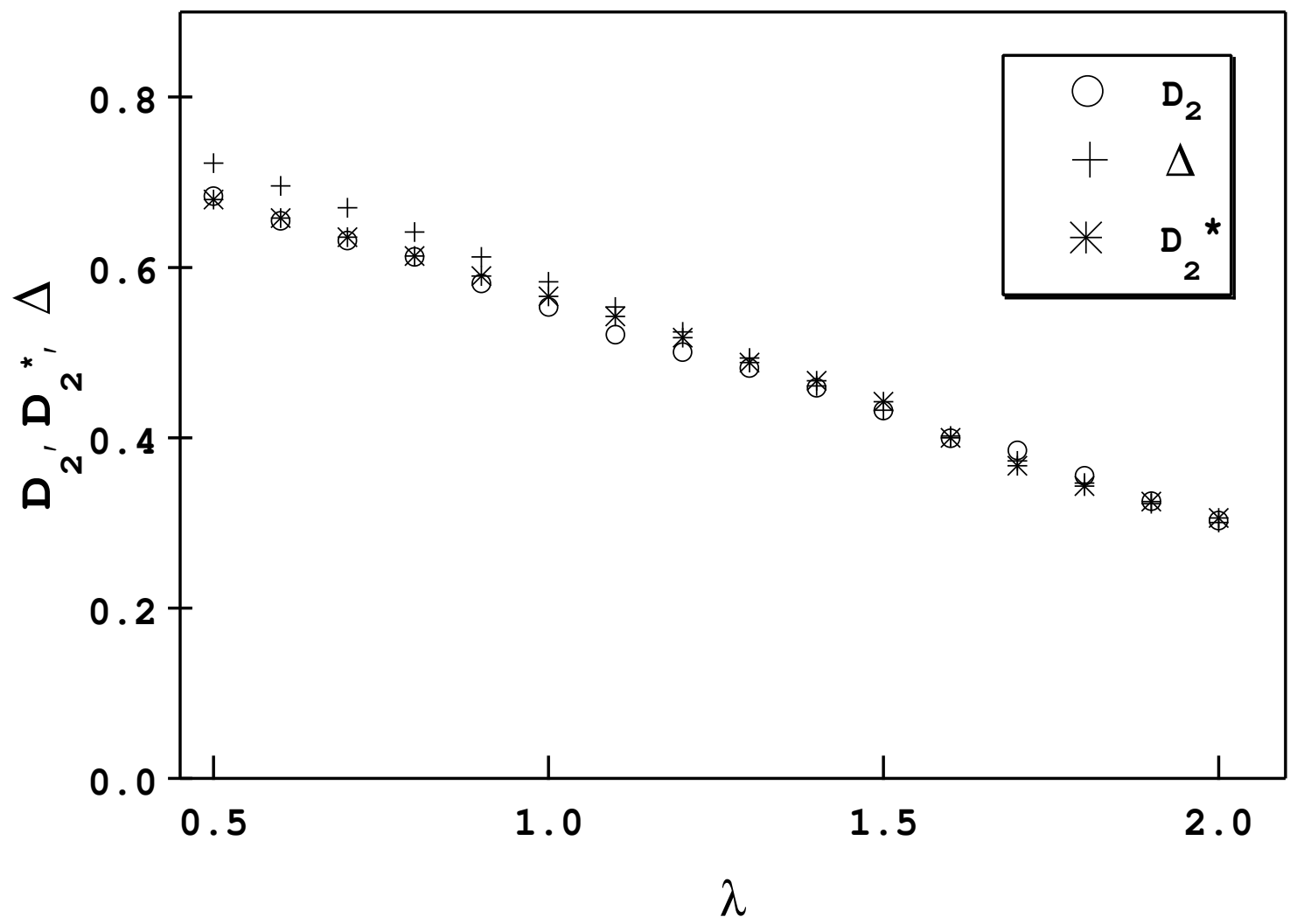

Figure 3 


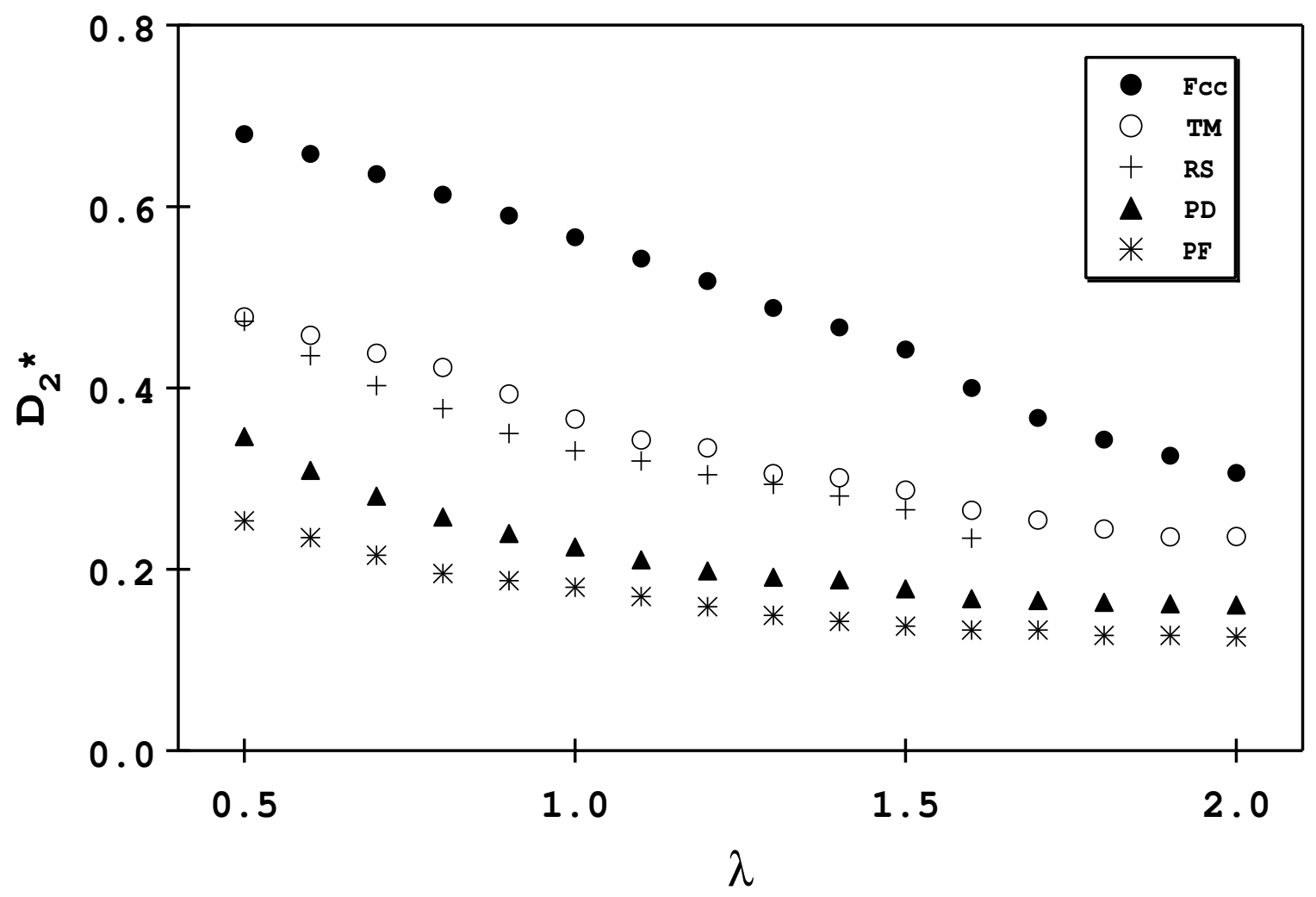

Figure 4 